\title{
Historia Magistra Vitae: Le topos de « l'Histoire comme maître de la vie » dans les controverses publiques sur la représentation de Soi et de l'Autre
} Historia Magistra Vitae: The Topos of History as a Teacher in Public Struggles over Self- and Other Representation

\section{Bernhard Forchtner}

Translator. Maria Brilliant

\section{(2) OpenEdition} Journals

Electronic version

URL: https://journals.openedition.org/aad/2170

DOI: $10.4000 /$ aad. 2170

ISSN: 1565-8961

\section{Publisher}

Université de Tel-Aviv

Electronic reference

Bernhard Forchtner, "Historia Magistra Vitae : Le topos de «l'Histoire comme maître de la vie » dans les controverses publiques sur la représentation de Soi et de l'Autre", Argumentation et Analyse du Discours [Online], 16 | 2016, Online since 09 April 2016, connection on 21 September 2021. URL: http:// journals.openedition.org/aad/2170 ; DOI: https://doi.org/10.4000/aad.2170

This text was automatically generated on 21 September 2021.

\section{(†)

Argumentation \& analyse du discours est mis à disposition selon les termes de la licence Creative Commons Attribution - Pas d'Utilisation Commerciale - Pas de Modification 4.0 International. 


\section{Historia Magistra Vitae : Le topos de « l'Histoire comme maître de la vie» dans les controverses publiques sur la représentation de Soi et de l'Autre}

Historia Magistra Vitae: The Topos of History as a Teacher in Public Struggles over Self- and Other Representation

\section{Bernhard Forchtner}

Translation : Maria Brilliant

\section{AUTHOR'S NOTE}

Ce texte est une version légèrement remaniée d'une contribution à Contemporary Critical Discourse Studies, sous la direction de Christopher Hart et Piotr Cam, et publié par Bloomsbury en 2014 (publiée en traduction, avec l'aimable autorisation de l'éditeur). Du fait qu'il y a eut certains malentendus à l'étape du copy-éditing, je saisis cette opportunité pour actualiser les figures 2, 3, 4, 7 et 9. Ainsi, cette version présente l'article tel qu'il était originellement prévu pour publication (bien que mes opinions sur le sujet, la méthode et son application aient évolué depuis, c'est intentionnellement que j'ai évité d'y apporter des modifications supplémentaires). Je suis reconnaissant aux responsables de Argumentation et Analyse du Discours et de ce numéro spécial de m'avoir offert cette possibilité de republier mon article corrigé en français. La recherche a été financée par des bourses d'études DOC allouées par l'Académie Autrichienne des Sciences, et par le Département de Linguistique et de Langue Anglaise à l'Université de Lancaster. Je remercie Ruth Wodak et Raimondo Frei pour leurs commentaires sur une 
version précédente de cet article. Toutes les erreurs me sont bien évidemment imputables.

\section{Introduction}

Dans le traité de Cicéron De Oratore (De l'Orateur) terminé en l'an 55 av. J.C., on peut lire la fameuse déclaration (1869, II, 9) selon laquelle l'histoire est « le flambeau de la vérité, l'âme du souvenir, l'oracle de la vie [littéralement : le maître de la vie : Historia Magistra Vitae]». De fait, on crut longtemps que l'étude du passé, en engageant à suivre son exemple, servirait de guide au présent. Dès lors, cependant, qu'on adopte une perspective axée sur les discours, les représentations et leurs conséquences, le centre de gravité se déplace. On cherche à savoir comment le topos de l'Historia Magistra Vitae (l'Histoire comme maître et guide), et l'affirmation qu'on connaît les «leçons » du passé, qu'on les a apprises et qu'on leur reconnaît le rôle de guide, confèrent du sens à des acteurs, des événements, des objets et des processus contemporains, qui distinguent un «nous » d'un « ils ». L'analyse du discours, et plus particulièrement son versant critique, soulève des questions comme : quels passés et présents sont définis par le topos de l'«Historia Magistra Vitae»? Quelles conclusions sont facilitées par l'utilisation de ce topos dans les controverses publiques sur la démarcation entre «nous » et " eux »? La pertinence de ces questions peut facilement être illustrée par deux citations tirées d'un débat concernant une attaque imminente sur l'Irak, au parlement européen, le 29 janvier 2003 :

Exemple 1: Des tourmentes politiques ont ravagé l'Europe au siècle dernier. L'obsession de pouvoir idéologiquement « légitimée » manifestée par des dictateurs a fait des millions de victimes. Ils n'ont d'ailleurs pas épargné leurs propres citoyens. Le parallèle avec le régime détestable de Saddam Hussein n'est-il pas évident ici ? [...] Les pays membres de l'Union Européenne sont-ils prêts à avoir cela sur la conscience, particulièrement au vu de leurs propres expériences douloureuses du siècle dernier ? [...] L'histoire européenne récente devrait montrer aux dirigeants gouvernementaux quelle est leur responsabilité à cet égard.

Exemple 2: L'Amérique devrait écouter la vieille Europe, cette vieille dame sage, couverte de larmes et de sang [...]. Que l'Amérique l'écoute! Cette vieille dame dirait : optez pour la sécurité à l'aide de la loi internationale, inclinez-vous devant les décisions des Nations Unies, acceptez une nouvelle réunion des Nations Unies, que nous devons exiger, en vue d'une nouvelle résolution.

2 Ces deux exemples illustrent non seulement les utilisations conflictuelles du topos de l'Histoire comme guide du présent, mais aussi les difficultés à juger de l'adéquation des conclusions auxquelles il nous mène. Tandis que la première citation tirée d'une déclaration de Bastiaan Belder, membre du parlement européen du groupe "L'Europe de la Liberté et de la Démocratie ", mobilise le passé dans le but de soutenir la guerre en Irak, le second orateur, Bernard Poignant, du Parti Socialiste Européen, utilise ce même passé afin de justifier son opposition à la guerre. Bien entendu, l'auditoire et les lecteurs pourront manifester leur préférence ; mais il semble bien qu'aucune des deux interprétations du passé et du présent ne puissent être purement et simplement rejetée. En d'autres termes, il est difficile d'identifier la « véritable nature » du passé, la leçon de l'Histoire, et je soutiens donc (de manière sans doute non controversée) que les analystes du discours devraient se concentrer sur la construction des acteurs et de leurs identités, des événements et de leur signification, des objets et de leur 
mobilisation, des processus et de leur sens, telle qu'elle s'effectue par et à l'aide de ce topos.

En conséquence, le présent article soulève la question de savoir comment l'Historia Magistra Vitae est utilisée dans les confrontations verbales qui portent sur les leçons « correctes » à tirer du passé et sur la construction des « autres » comme fourvoyés. Je suis donc intéressé par le caractère persuasif de l'usage de la langue, et me propose d'étudier les moyens propres à persuader (Aristote 2003: 22) relatifs à ce topos particulier. Plus spécifiquement, je conceptualiserai quatre utilisations possibles du topos en question, basées sur des prémisses différentes qui mèneront à des conclusions différentes. En bref, j'examine les modalités selon lesquelles des récits du passé sont la toile de fond qui permet de plaider en faveur de leçons portant sur le présent. Néanmoins, au lieu d'inclure des références sur les leçons tirées de souvenirs d'héroïsme et de gloire nationale, ces conceptualisations se limiteront ici aux leçons tirées de méfaits du passé, tels que l'Holocauste comme paradigme du crime contre l'humanité. Je vais donc conceptualiser et illustrer ce que j'appelle la «rhétorique du jugement», la "rhétorique de l'échec», la "rhétorique de la repentance» et la "rhétorique du jugement-repentance $»^{1}$.

4 En premier lieu, la rhétorique du jugement aide à intégrer des souvenirs collectifs dans une identité collective en indiquant des méfaits passés qui n'ont pas été commis par le "groupe intérieur » (in-group), lequel affirme néanmoins avoir assimilé les «leçons » actuelles du dit méfait, et en conséquence rejette des acteurs et actions du groupe extérieur (out-group) présentés comme n'étant pas au fait de ces leçons. Les arguments de pacification en constituent probablement l'exemple le plus probant, et ont figuré au premier plan des confrontations publiques sur la Guerre Froide et la Guerre d'Irak en 2003, par exemple. Ces arguments identifient un méfait passé (« des pacifistes naïfs croyaient pouvoir apaiser Hitler »), en tirent les leçons (« il vaut mieux stopper les dictateurs tôt que tard»), et ciblent un "autre » extérieur (« de nos jours, la gauche essaye encore une fois d'apaiser... »). Deuxièmement, la rhétorique de l'échec met elle aussi l'accent sur un méfait passé qui n'a pas été commis par le in-group en question. Toutefois, l'affirmation d'avoir tiré la leçon qui s'impose est à présent dirigée contre le groupe intérieur, qui est appelé à faire son examen critique à la lumière de ce passé. Des références à des événements passés universellement dénoncés comme des méfaits, entre autres l'Holocauste et la Guerre du Vietnam, abondent dans ce genre de rhétorique. En troisième lieu, la rhétorique de la repentance dénonce le caractère négatif de notre passé et met, plus ou moins explicitement, l'accent sur la nécessité constante de démarquer le groupe intérieur actuel de ses actions coupables précédentes. L'actuelle vague d'excuses publiques issues d'Etats et d'organisations nongouvernementales, entre autres le discours du Président français François Hollande (2012) étudié ci-dessous, commémorant la détention et la déportation de Juifs français en 1942, fait usage d'une telle rhétorique. Bien que de tels actes de langage soient souvent problématiques, ils ne sont pas en soi le signe d'une tendance à la mode; ils permettent une reconstruction potentielle, plus inclusive et ouverte, d'identités communes. Les rhétoriques du jugement, de l'échec et de la repentance sont des structures familières dans les discours sur le passé. Par contraste, je me tourne en quatrième lieu vers la dernière œuvre publiée d'Albert Camus, La Chute (1956), afin de proposer une conceptualisation originale de la rhétorique du jugement-pénitence ${ }^{2}$. Ici, l'affirmation d'avoir appris les leçons du passé est basé sur les aveux du groupe 
intérieur qui avoue avoir mal agi, afin de «nous » présenter comme étant moralement supérieurs face à un « autre » extérieur.

5 Se concentrer sur les utilisations du topos de l'Historia Magistra Vitae semble aujourd'hui d'autant plus opportun que les dernières décennies ont considéré les souvenirs du passé - et en particulier celle des méfaits passés - comme "remplaçant le progrès et les révolutions pour devenir les métaphores-maîtresses de l'Histoire» (Giesen $2004: 10$ ). $\mathrm{Au}$ cours de cette évolution, la mémoire en est venue à devenir une "nouvelle religion séculière» (Müller 2002 : 19). C'est donc le contexte spécifique de notre temps, «l'âge des excuses " (Gibney et al. 2008), qui permet une vaste exploitation de l'Historia Magistra Vitae. De plus, la construction discursive d'identités communes, et plus particulièrement la construction d'identités nationales dans leur rapport au passé, a constitué l'un des domaines primordiaux des études critiques du discours ${ }^{3}$. Ces études se focalisent principalement sur les constructions traditionnelles, héroïques et complaisantes d'événements passés, et sur l'antisémitisme et le chauvinisme qui en découlent. Cependant, on trouve également dans ces constructions des emplois de l' Historia Magistra Vitae qui servent à structurer des identités particulières, et à en légitimer les positions.

Le profit que l'on peut tirer de la conceptualisation que je présente ici est double: premièrement, en proposant une reconstruction abstraite des utilisations de l'Historia Magistra Vitae, j'en offre une taxinomie globale. Bien évidemment, des exemples empiriques ne pourront jamais être similaires à des types purs; néanmoins, leur reconstruction éclairera leur fonctionnement et leurs fonctions. D'autre part, et je ne l'indique qu'en passant, ces types peuvent servir à renforcer une perspective critique en mettant à nu un mécanisme social susceptible de dé/bloquer des processus d'apprentissage collectif, c'est-à-dire en traçant des frontières symboliques plus ouvertes /fermées de la communauté respective.

7 La seconde partie clarifie les notions de discours, de (mnémo) histoire, et de «narrativisation» (White 1980) dans leurs rapports entre elles, et dans leur rapport aux études critiques de discours (CDS), en particulier à l'approche historique du discours (DHA)4. Suivra une discussion sur le concept de topos en général, et du topos de l'Histoire en tant maître de vie en particulier. En se fondant, entre autres, sur le schéma argumentatif de Stephen E. Toulmin, la quatrième partie conceptualise et illustre ce topos en exposant les rhétoriques du jugement, de l'échec, de la repentance et du jugement-pénitence. Je conclus par un bref résumé des résultats, et une réflexion sur la possibilité d'une évaluation critique de ces quatre genres, en y ajoutant quelques remarques finales sur le problème que soulève la démarcation entre ces rhétoriques.

\section{Du discours, de la (mnemo)histoire, et de la narrativité à...}

8 Dans ce qui suit, je m'appuie essentiellement sur la DHA et les CDS, bien que les points principaux articulés dans cet article ne se limitent pas à leur cadre. Ceci concerne en particulier les concepts de discours et contexte, dont je vais d'abord traiter avant de me tourner vers des concepts théoriques supplémentaires.

9 A la suite de Reisigl et Wodak (2009: 89), je considère le discours comme un agglomérat de pratiques sémiotiques dépendantes de leur contexte, reliées à une macro-topique ; 
comme étant « socialement constituées et socialement constituantes » et comme " liées à l'argumentation de prétentions à la validité [...], qui impliquent plusieurs acteurs dont les points de vue sont différents ». Les discours ne sont donc pas considérés comme étant "à perspective unique ", à la différence d'autres approches des CDS (Reisigl 2013 : 79) : ils sont définis par leur lien au thème discuté, par exemple les discours sur la guerre en Irak. L'avantage d'une notion aussi fortement axée sur l'empirie est, de toute évidence, qu'elle permet de déceler des positions opposées à l'intérieur des discours, entre autres des affirmations conflictuelles sur les «leçons » du passé. Ces affirmations construisent des liens intertextuels et interdiscursifs variés avec d'autres textes et discours (Reisigl et Wodak, 2009: 90-93), ce qui signifie que la semiosis dépend amplement du contexte. La notion de contexte de la DHA comporte quatre dimensions ; elles vont du co-texte aux liens intertextuels et interdiscursifs, au «contexte de situation » spécifique, et au contexte socio-politique et historique plus large (ibid.: 93). Par conséquent, connaître le contexte historique est primordial pour l'analyse historique du discours pour comprendre (et éventuellement évaluer de façon critique) le sens et sa (re-)production.

10 L'accent mis sur le contexte historique est donc crucial. Toutefois, du fait que l'analyse du discours s'intéresse moins au passé en lui-même qu'à ses utilisations, la DHA devrait être (et généralement est) moins intéressée par la notion positiviste de l'Histoire et de la vérité historique, que par ce que Jan Assmann (1997: 8-9) appelait la "mnémohistoire »: " contrairement à l'histoire à proprement parler, la mnémo-histoire ne se penche pas sur le passé en tant que tel, mais uniquement sur le passé tel qu'on s'en souvient ». Afin de comprendre les luttes publiques pour la légitimité, ainsi que les processus discursifs qui y sont liés dans le but de démarquer le «nous » du « eux », il est donc nécessaire de comprendre les mémoires collectives ${ }^{5}$. Celles-ci ne sont pas un agrégat de mémoires individuelles au sein d'un sujet collectif quelconque, mais bien le cadre social dans et par lequel des individus se souviennent d'événements passés. Maurice Halbwachs (1985: 31) a très bien montré que « chaque mémoire individuelle était un 'point de vue' sur la mémoire collective », en soulignant leur construction sélective et leur fonction primordiale dans la garantie d'un sentiment de continuité communautaire.

11 En tant que telles, les mémoires collectives doivent être considérées comme des récits qui circulent dans des réseaux spécifiques, délimitant ainsi des groupes (Tilly 2002). Je plaide donc pour une incorporation plus explicite de l'analyse narrative, et en particulier de l'analyse structurale des récits, au sein des $\mathrm{CDS}^{6}$. Semblable incorporation de la narratologie n'est pas seulement importante d'un point de vue empirique; elle concerne aussi la question de la (mnémo)histoire en analyse du discours. En effet, c'est dans et par des récits que les arguments sont avancés. C'est-à-dire que si les récits utilisent des arguments, ils servent aussi eux-mêmes d'arguments pour des genres particuliers d'actions. En outre, le récit constitue une catégorie ontologique clé. C'est surtout Paul Ricoeur (1988 : 241) qui établit que la temporalité ne peut s'exprimer dans le discours direct de la phénoménologie, mais exige la médiation du discours indirect de la narration. En dépit de la manière dont Ricoeur interprète le discours, le récit devient ici le principe organisateur de la vie sociale (cf. aussi Barthes 1977, White 1980). C'est ainsi que toute référence au passé passe par la médiation d'une forme narrative, ce que Hayden White définissait comme la «narrativisation» du passé. De telles narrativisations traitent du choix des événements - un événement étant défini comme une action causant un changement de l'état des choses - et du choix d'organiser les 
événements selon le schéma fondamental: début (état antérieur) - milieu (l'événement) - fin (nouvel état). La force du récit provient précisément de la pratique qui consiste à arranger des événements (sélectionnés) en une intrigue unifiée, comprenant la conclusion de cette séquence. En d'autres termes, comme le dit van Dijk (1980: 14): " a 'good story' is one which has a "point" ». De même, White (1980: 24) discute du «sens moral» transmis par tout récit, du fait que l'acte même de narrativisation - et non le passé lui-même - sélectionne et organise des événements historiques de manière particulière. Dans le même ordre d'idées, Ricoeur (1988: 249) indique qu'aucun récit ne peut être éthiquement neutre. Cela ne signifie pas que l'on ne puisse prouver la véracité factuelle d'événements historiques ; pourquoi ne pourrait-on dire ce qui est arrivé le 9 novembre 1989 (la chute du mur de Berlin) ? Il s'agit plutôt du fait que les événements qui ont eu lieu dans le passé ne conditionnent pas leur représentation dans le présent; il s'agit là d'un processus qui dépend de leur agencement narratif nécessairement sélectif dans ce même présent. Ceci nous ramène à la compréhension de l'Historia Magistra Vitae, ainsi qu'à la fonction de l'acte de reconstruction du contexte historique d'un discours particulier. L'analyse doit non seulement être consciente de l'inhérente et irréductible mise en perspective qu'effectue la reconstruction historique, mais aussi - et c'est la raison pour laquelle on débat du message moral transmis par tout récit - elle doit évacuer l'idée de «leçons objectives ». L'attention doit au contraire être dirigée vers ce à quoi tendent ces récits, et la manière dont ils le réalisent linguistiquement.

\section{Le topos de l'Historia Magistra Vitae}

D'un point de vue historique, l'étude des mémoires, c'est-à-dire de la substance des frontières narratives, et la rhétorique sont intimement liées. Tandis que l'art de la mémoire fut probablement perfectionné au Moyen Age, Cicéron (1959, II, $84: 352$ ) indiquait déjà que "la science mnémotechnique " remontait au poète lyrique grec Simonides de Ceos (556-468 av. J.C.). On prétend que Simonides aurait identifié les participants d'un banquet - que lui-même avait quitté juste avant qu'ils ne fussent écrasés par un toit qui s'était effondré sur eux - à l'aide de l'agencement bien ordonné de sa mémoire. Il en déduisit que " pour exercer cette faculté, il faut [...] imaginer dans sa tête des emplacements distincts, et y attacher l'image des objets dont on veut garder le souvenir $»^{7}$. Mémoriser un discours signifiait donc passer à travers une série de « lieux », ou topoi (Cicéron utilisait le terme latin loci).

Dans les travaux d'Aristote, ces topoï font partie à la fois de la dialectique et de la déduction rhétorique; dans le schéma aristotélicien complet de la rhétorique, ils se relient à l'étape qui consiste à trouver des arguments - heuresis ou inventio (Kienpointer 1995 : 454). Pour Aristote (1982, I, $2: 8$ ), un topos dans un syllogisme rhétorique indique la présence d'une prémisse essentielle, bien que généralement implicite, dont dépendent les performances persuasives (ibid., I, 2, 21-22). Tandis qu'Aristote considérait les topoï essentiellement comme des éléments abstraits ou généraux (par exemple le topos du plus ou moins qui peut se concrétiser ainsi : "si les dieux euxmêmes ne savent pas tout, c'est encore moins probable pour les hommes » (I, 23, 4). A l'inverse, Cicéron proposait le concept de locus communis. De tels loci constituent des arguments matériels, "tout prêts " (Rubinelli 2009: 106), qui ne peuvent être utilisés que dans des contextes ou des cas comparables. 
14 De même, Toulmin a introduit le concept de "garantie " pour désigner les moyens confirmés de liaison entre les données (la base sur laquelle l'affirmation ou la conclusion se fonde) et la conclusion (le point d'arrivée) dans des domaines spécifiques. Les garanties sont des «statements indicating the general ways of arguing being applied in each particular case and implicitly relied on as ones whose trustworthiness is well established » (Toulmin et al. 1979: 43). Martin Wengeler (2003: 67) considère les topoï comme des "figures de pensée utilisées dans l'approche d'un problème politique ». Dans cette ligne d'argumentation, mais plus ouverts en ce qui concerne la définition des topoï en tant que formels ou matériels, Reisigl et Wodak (2001: 74-75, cf. aussi Kienpointer $1995: 454)$ considèrent les topoï comme des règles qui relient les données aux conclusions. Ils sont

des parties de l'argumentation hautement formalisées qui font partie des éléments obligatoires de l'argumentation, et prennent la forme de prémisses soit explicites, soit à inférer. Ce sont des garanties ou des « règles de conclusion » plus ou moins formelles (par exemple locus a minore) ou bien liées à un contenu (topos des contraintes extérieures), qui relient un argument ou des arguments à une conclusion, une affirmation. En tant que tels, ils justifient le passage d'un argument, ou d'arguments, vers la conclusion (Wodak et al. 1999 : 34).

Pour résumer, et bien que les avis soient partagés quant à leur caractère formel (présenté de manière paradigmatique par Aristote), ou bien matériel (présenté de manière paradigmatique par Cicéron), les topoï sont toujours considérés comme des matériaux de construction auxquels les acteurs doivent avoir recours afin de persuader (ou convaincre) l'auditeur, c'est-à-dire afin d'adapter avec succès leur performance aux connaissances partagées par l'auditoire.

Revenant au topos de l'Historia Magistra Vitae, l'Histoire comme maître de vie, nous nous tournons à nouveau vers De l'Orateur de Cicéron et à la déclaration $(1869$, II, 9) selon laquelle l'histoire est «le flambeau de la vérité, l'âme du souvenir, l'oracle de la vie [Historia Magistra Vitae]». Reinhart Koselleck (2004: 36) prétendait qu'en ce qui concerne l'historiographie, ce topos a longtemps présenté la mémoire du passé comme devant guider les actions contemporaines et permettre de se comprendre soi-même, mais qu'il semble avoir disparu durant la période des Lumières qui a découvert «l'unicité des processus historiques, et la possibilité du progrès ». Selon Koselleck, l'ascension de la modernité a sonné le glas de certitudes antérieures enracinées dans un espace chrétien d'expériences et dans son horizon d'attente - rendant ainsi le topos obsolète. Ceci ne signifie pas, toutefois, que son utilisation rhétorique dans des drames politiques n'ait pas prévalu. Or, à ma connaissance, il n'existe pas d'étude récente qui se serait penchée plus particulièrement sur l'usage public de ce topos. Il est toutefois intéressant de constater que Walther Kindt (1992: 202) se réfère brièvement à l'Historia Magistra Vitae dans son analyse de textes allemands portant sur la guerre d'Irak de 1991/2, indiquant que ce topos pourrait faciliter la représentation négative de « l'autre »- comme borné et ignorant. Une analyse plus pertinente pour ce sujet est le travail de Wodak et al. (1998: 205 ; 2009: 86 ; cf. aussi Reisigl et Wodak 2001: 80), sur l'identité nationale autrichienne, qui reconnait le topos comme «minimisant et banalisant le passé », du fait qu'il sert de "zone-tampon collective, cognitive et émotionnelle » à travers laquelle le groupe intérieur perd en dernière instance de vue les victimes. De ce fait, ces auteurs (ainsi que d'autres) ont analysé des usages particuliers de ce topos sans pour autant en élaborer une typologie plus abstraite. 
Par contre, ce que Reisigl et Wodak présentent est une définition nette du topos de l'Histoire (ils considèrent le topos de l'Historia Magistra Vitae comme une sous-catégorie du topos de l'Histoire). Dans ce qui suit, pour traiter de l'Histoire comme maître et guide de la vie, je reprendrai leur définition du topos de l'Histoire :

Du fait que l'Histoire enseigne que des actions spécifiques ont des conséquences spécifiques, on devrait accomplir ou omettre d'accomplir une action spécifique dans une situation spécifique (prétendument) comparable à l'exemple historique auquel on se réfère (ibid. : 80).

En transposant la définition de Reisigl et Wodak sur un modèle simplifié de Toulmin, la reconstruction suivante émerge (Figure 1). En racontant une action particulière passée (A1, les données), la garantie (ou topos) de l'Histoire comme guide de la vie justifie la conclusion, c'est-à-dire le fait d'agir de certaine manière (A2) pour obtenir un certain résultat (X2). Ce schéma argumentatif peut s'appliquer aussi bien à des microarguments qu'à des unités plus importantes, voire même à des récits complets.

$\begin{aligned} & \text { Une action } \mathrm{A}_{1} \text { a eu des } \\ & \text { conséquences bénéfiques / terribles } \\ & \text { dans le passé }\left(\mathrm{X}_{1}\right) .\end{aligned}$
$\begin{aligned} & \text { avons appris les leçons, nous devrions } \\ & \text { accomplir une action } \mathrm{A}_{2}(\text { similaire } \\ & \left.\text { à } \mathrm{A}_{1} \text { mais différente de } \mathrm{B}_{2} \text { ou } \mathrm{C}_{2}\right) \\ & \text { afin d'obtenir } \mathrm{X}_{2} .\end{aligned}$

Du fait que l'histoire enseigne que des actions spécifiques ont des conséquences spécifiques, on devrait accomplir ou omettre d'accomplir une action spécifique dans une situation spécifique (prétendument) comparable à l'exemple historique auquel on se réfère.

Figure 1 : Reconstruction du topos de l'histoire en tant qu'enseignant.

\section{Le passé dans le présent : conceptualisation de quatre utilisations de l'Historia Magistra Vitae}

Après avoir traité de ce topos, je me tourne maintenant vers ses utilisations. M'inspirant du modèle de Toulmin exposé ci-dessus, je vais conceptualiser et traiter quatre exemples afin d'illustrer la réalisation empirique des rhétoriques du jugement, de l'échec, de la repentance et du jugement-pénitence. Ces quatre exemples ne sont présentés qu'à titre d'illustration et sont tirés de différents contextes - le Danemark en 2003, Le Royaume-Uni en 2009, la France en 2012 et enfin l'Europe en général; cependant, dans tous les cas, le méfait passé facilite l'accès au " point crucial » obtenu par ces interventions. Ce faisant, je m'inspire de la notion de la DHA sur les stratégies discursives (Reisigl et Wodak, 2009: 94) en tant qu'actes plus ou moins conscients ou automatisés, et indique leurs réalisations linguistiques, qui permettent des représentations particulières de soi et des autres. Je vérifie ainsi les moyens par lesquels les désignations et les prédicats (ainsi que les atténuations et/ou intensifications et mises en perspective) des acteurs, des événements, des objets et des processus sont reliés entre eux et forment le socle des arguments. 


\subsection{La rhétorique du jugement}

\section{mentionné ci-dessus, le topos relie les données (un méfait passé commis par un groupe extérieur) à la conclusion (que des actions similaires proposées actuellement par d'autres devraient être évitées). Ainsi, bien que la rhétorique du jugement ne nie pas qu'il y ait eu méfait dans le passé, elle n'en adopte pas pour autant une position critique envers ce passé comme étant «le nôtre », ni même comme ayant une pertinence en ce qui concerne «notre » présent. De ce fait, la rhétorique du jugement se fonde sur une double exclusion, à la fois de «notre » méfait passé (ou du moins de sa mise en lumière ou de son occultation), et de « l'autre ", qui n'a pas assimilé les leçons du passé (Figure 2). Tout en ne problématisant pas «notre "passé, on n'en revendique pas moins l'enseignement fourni par «le» passé, dans le but de délégitimer un «autre» extérieur, ainsi que ses actes.}

vie réside probablement dans ce que j'appelle la rhétorique du jugement. Comme

\begin{tabular}{l|l}
$\begin{array}{l}\text { Un méfait terrible a été } \\
\text { commis par d'autres dans le passé. }\end{array}$ & $\begin{array}{l}\text { les leçons de l'histoire tandis que d'autres } \\
\text { ne les ont pas apprises. }\end{array}$ \\
\end{tabular}

Du fait que l'histoire enseigne que des actions spécifiques ont des conséquences spécifiques, on devrait accomplir ou omettre d'accomplir une action spécifique dans une situation spécifique (prétendument) comparable à l'exemple historique auquel on se réfère.

Figure 2 : Une reconstruction de la rhétorique du jugement.

Pour illustration, je prends l'exemple du Danemark au cours du débat sur la guerre d'Irak en $2003^{8}$. De même que dans d'autres pays (non-)européens, des débats houleux sur la guerre amenèrent un gouvernement de centre-droite présidé par le Premier Ministre Anders Fogh Rasmussen à rejoindre la «Coalition des Volontaires ». Cette position était initialement justifiée, en raison du danger apparent que présentaient des armes irakiennes de destruction massive. Cet argument s'étant effondré vers la mi-2003, Rasmussen légitima de plus en plus la participation danoise à la guerre d'Irak en liant celle-ci à une critique de la politique de collaboration menée par son pays avec l'Allemagne nazie entre 1940 et 1943 . Ce faisant, il contredisait le narratif danois d'après-guerre selon lequel les nationaux avaient héroïquement résisté aux occupants allemands. En réalité, l'élite politique et économique danoise (ainsi que de grandes parties de la population générale) avaient coopéré avec l'Allemagne, collaboration qui ne cessa qu'en 1943 en raison d'un soulèvement populaire.

Je traite ailleurs (Forchtner 2013) des subtilités du discours de Rasmussen prononcé en août 2003. Je voudrais cependant signaler ici les moyens rhétoriques principaux, sans doute moins sophistiqués, utilisés par ses partisans. L'extrait suivant est tiré d'un article de journal rédigé par le Ministre de la Culture (alors Conservateur) Brian 
Mikkelsen (2003), intitulé « Nous ne devons plus jamais tomber dans le piège de la neutralité » :

nous avons subi une véritable épidémie de neutralité social-démocratique-libérale et avons appliqué une sorte de politique de l'autruche universelle, par lesquelles le mouvement pour la paix a réussi à mettre un bandeau sur les yeux de nombreux jeunes de bonne volonté.

Actuellement, en particulier lorsqu'on se remémore avec respect le mois d'août 1943, nous devons nous rappeler les uns aux autres qu'il nous incombe d'éviter l'écueil de la neutralité, et l'action consistant à esquiver les dictateurs et despotes comme des œufs pourris, dont nous nous détournons. Au contraire, nous devons nous élever contre eux, et continuer à le faire encore et toujours. C'est l'esprit de 1943 qui doit éclairer notre route, et non celui de 1940 ou de 1968.

Le passé, de même que le présent, continuera à se jouer de nous. C'est pourquoi il est si important de continuer à nous souvenir et remémorer aussi bien et aussi précisément que possible. Car nous ne sommes pas à la merci de l'Histoire. L'Histoire n'est pas plus forte que nous. Nous devons simplement la respecter - et l'utiliser. Chaque nouvelle génération doit ainsi passer le flambeau du souvenir à la suivante. Ceci est la seule manière de combattre l'oubli, et de réduire le risque de répéter des erreurs qui auraient pu être évitées. est renforcé par les moyens linguistiques utilisés. Pour peu que l'on porte son attention sur la désignation et le prédicat, on voit qu'un gouffre sépare le groupe intérieur non spécifié mais homogène, du groupe extérieur négatif, plus spécifiquement désigné. Le premier se construit par l'usage répétitif d'expressions déictiques telles que "nous » (sujet et complément) qui se rapportent à la «bonne » partie du peuple danois, du fait que l'article a paru dans un journal national (dont le lectorat est essentiellement bourgeois). Le gouffre s'élargit encore plus par la représentation de cet acteur social comme étant soit la victime passive de "l'autre: («nous avons souffert»), soit activé déontologiquement par «devons»/«devrions». Similairement, et concernant la manière dont ce groupe intérieur est lié à des événements ou processus, les conséquences de la guerre - telles la violence et la souffrance (civile) - sont à peine effleurées dans le texte (hormis une référence aux « morts tragiques » et au " fanatisme et à la soif de sang " des terroristes). Par contre, ce qui devrait être accompli est dépeint dans un vocabulaire qui échappe à la controverse et dans des métaphores ludiques, telles qu'esquiver les œufs pourris, et éclairer notre route. Cela ne sert pas seulement de toile de fond à la question de savoir ce que les politiques de «l'esprit de 1943 » pourrait bien suggérer de nos jours; est évoquée également une délivrance, et l'affranchissement par rapport à un sombre passé - dont, toutefois, « nous » (le groupe intérieur confirmé de «bons Danois ») n'est pas responsable.

Par contre, «l'autre ", et les événements et processus qui lui sont liés, sont décrits de façon beaucoup plus spécifique. En premier lieu, ces acteurs sont identifiés à l'aide d'anthroponymes idéologiques (tout au moins, ces termes sont susceptibles de recevoir 
une connotation négative par les lecteurs de ce journal et par la clientèle du gouvernement): "la gauche", "les libéraux », "le mouvement pour la paix». Ce dernier terme est particulièrement intéressant du fait que l'auteur avance un argument afin de justifier son inclusion dans la catégorie des «méchants». Mikkelsen utilise en effet le topos (ou le sophisme) d'une jeunesse innocente et naïve, expliquant le rejet de la guerre exprimé par de nombreux jeunes endoctrinés par la capacité de séduction du mouvement pour la paix (le fait que des arguments anti-guerre se réfèrent souvent à des manifestations de masse et à l'opinion populaire a pu être à l'origine de cette affirmation de Mikkelsen). En mentionnant 1968 (une autre désignation "idéologique»), une référence intertextuelle supplémentaire vise la gauche et les libéraux. Après tout, le Danemark a aussi été le théâtre d'une série de débats dans les années 1960 en raison, par exemple, de l'émergence de la Nouvelle Gauche (mais aussi de groupes libéraux variés). Ces débats portaient sur les initiatives concernant les armes anti-nucléaires, la critique de l'appartenance à l'OTAN et de la guerre américaine au Vietnam - qui avaient tous suscité des conflits avec le parti conservateur (DISS : 2005). En second lieu, les événements et processus sont présentés au lecteur à travers les termes, par exemple, d'« épidémie ", d'« écueil de la neutralité », de " la politique de l'autruche ", ou encore l'expression « esquiver les dictateurs ", qui comportent tous des connotations négatives. L'argumentation transforme cette interprétation en une connaissance historique fiable et "vraie ", par l'appel à « nous souvenir et remémorer aussi bien et aussi précisément que possible ", faisant ainsi du rejet des données un acte immoral et irrationnel.

Sur cette toile de fond, le topos de l'Histoire comme maître et guide relie les données selon lesquelles des erreurs passées ont été accomplies (et le sont encore) par la gauche et les libéraux, à la conclusion - énoncée implicitement dans cet extrait mais plus explicite dans des parties précédentes de l'article - qu'éviter ce genre d'erreurs exige de mener une action ferme contre Saddam Hussein (Figure 3). En accord avec Kindt, ce genre d'intervention dans des luttes publiques affirme que "nous» connaissons les leçons véritables de l'Histoire, dans un essai de délégitimer «l'autre » et ses actions, dénoncées comme relevant de l'obstination et moralement inférieures.

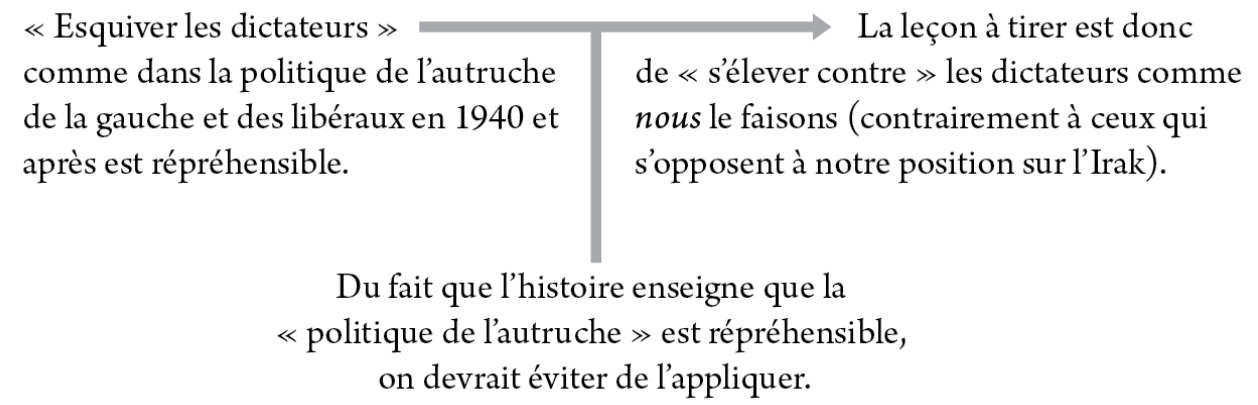

Figure 3 : La rhétorique de jugement de Mikkelsen.

\subsection{La rhétorique de l'échec}

Par opposition à la manière traditionnelle de relier des méfaits passés et présents, et la double externalisation par laquelle elle s'opère dans la rhétorique du jugement, je me tourne à présent vers une forme d'utilisation du passé plus autocritique. Dans la 
rhétorique de l'échec, les méfaits passés (les données) restent le fait d'un groupe extérieur ; néanmoins, la leçon qui en est prétendument tirée, la conclusion, n'est plus dirigée contre un «autre » extérieur, mais sert au groupe intérieur d'avertissement à ne pas répéter ces échecs. Connaître les leçons et tirer l'enseignement du passé exige un regard critique sur « notre » méfait actuel (ou potentiel), c'est-à-dire une vigilance constante du groupe intérieur.

Un méfait terrible a été Ainsi, nous ne devons pas commis dans le passé. commettre (prétendument) des méfaits comparables et en répéter les conséquences néfastes, afin d'éviter notre chute (potentielle).

Du fait que l'histoire enseigne que des actions spécifiques ont des conséquences spécifiques, on devrait accomplir ou omettre d'accomplir une action spécifique dans une situation spécifique (prétendument) comparable à l'exemple historique auquel on se réfère.

Figure 4 : une reconstruction de la rhétorique de l'échec.

L'extrait suivant est tiré d'un article rédigé par le journaliste britannique Robert Fisk (2009) dans le journal du Royaume-Uni The Independant. Cet article, qui traite de l'intervention militaire en Afghanistan de l'après-11 septembre, s'intitulait "L'Amérique joue son rôle habituel de soutien à un dictateur " et a été publié le 4 novembre 2009.

Pourrait-il y avoir une description plus exacte du message de félicitations d'ObamaBrown à Hamid Karzai d'Afghanistan, élu frauduleusement? D'abord, les Palestiniens ont tenu des élections honnêtes en 2006, ont voté pour le Hamas et en furent - et le sont encore - brutalement punis, puis les Iraniens ont tenu des élections frauduleuses en juin qui ont remis le bizarre Mahmoud Ahmadinejad au pouvoir, alors que tout le monde hors d'Iran (et beaucoup à l'intérieur) le considèrent comme un dictateur. Mais à présent nous avons Karzai le vénal, le corrompu, le sectaire au pouvoir après des élections encore plus vastement truquées que la version iranienne, et - hop, nous l'aimons chèrement et acceptons son élection totalement frauduleuse. [...]

La Marche Folle de l'Histoire était le titre choisi par Barbara Tuchman pour son ouvrage sur les gouvernements - de Troie à l'Amérique de l'ère du Vietnam - qui appliquent des politiques contraires à leurs propres intérêts. Et nous ferions bien de nous souvenir de l'histoire du Vietnam. Ainsi que l'indiquait hier Patrick Bury, un vétéran britannique de notre aventure afghane actuelle, l'exemple du Vietnam est en l'occurrence tout à fait pertinent.

Déjà en 1967, les Américains ont supervisé une élection "démocratique " au Vietnam qui a offert la présidence à l'ex-général corrompu Nguyen Van Thieuman [sic!]. Dans une élection frauduleuse que les Américains ont déclaré être "dans l'ensemble honnête » - il reçut 38 pour cent des voix - les opposants de Thieu n'avaient pas accepté de se présenter contre lui parce que l'élection était une farce. $[\ldots]$

Mais tout ceci fait partie d'un modèle sinistre. Les forces américaines prenaient part à une guerre civile au Vietnam tout en déclarant qu'ils soutenaient la démocratie et la souveraineté du pays. Au Liban en 1982, ils déclaraient soutenir le président élu « démocratiquement » Amin Gemayel et prirent le parti des Chrétiens 
maronites dans la guerre civile. Et maintenant, après des élections de Disneyworld, ils se placent du côté du gouvernement-Karzai contre les villageois de Pashtun au sud de l'Afghanistan, où vivent les Talibans. Où est le prochain My Lai ? Les journalistes devraient éviter d'énoncer des prédictions. Dans ce cas-ci, je ne suivrai pas ce conseil. Notre mission occidentale en Afghanistan se soldera par un désastre absolu.

29 des méfaits passés commis par "d'autres». On se réfère constamment ici aux actions liées à «l'Amérique à l'ère du Vietnam » comme étant l'événement initial duquel les acteurs auraient dû tirer des leçons. Que les actions américaines commises soient narrées de façon négative ressort clairement du fait qu'elles sont liées, parmi d'autres, à «La Marche folle de l'Histoire", au soutien "américain » pour un «ex-général corrompu » en 1967, à une "élection frauduleuse » approuvée par « les Américains ", un «modèle sinistre » de "forces américaines » impliquées dans des guerres civiles, ainsi qu'à une référence au massacre de My Lai. Le second paragraphe établit explicitement le sens de la guerre du Vietnam («Et nous ferions bien de nous souvenir de l'histoire du Vietnam »). Ceci est renforcé par le « rapport d'un soldat britannique vétéran » (argumentum ad verecundiam) qui a signalé la pertinence actuelle de la guerre du Vietnam. Il est important de noter, et j'y reviendrai, que cette autorité britannique caractérise le méfait actuel, à savoir la guerre en Afghanistan, comme «notre [...] aventure $»^{10}$.

Nous tournant à présent vers le méfait présent, la référence à "Obama-Brown » (le Président d'alors des Etats-Unis Barack Obama et le Premier Ministre du Royaume-Uni de l'époque Gordon Brown) et aux "gouvernements", qui sont critiqués pour leur hypocrisie dans leur soutien à Hamid Karzai sans souci de leurs intérêts propres respectifs, pourrait suggérer une faille entre un groupe intérieur («les innocents») et une élite politique "moralement corrompue ", seule responsable de la répétition des erreurs passées. En outre, les Etats-Unis sont crédités de leur « rôle habituel de soutien à un dictateur » et, dans le dernier paragraphe, Fisk indique qu'ils « ont pris le parti du gouvernement de Karzai ». Quoi qu'il en soit, et bien que la rhétorique du jugement soit activée dans cet article puisque les Etats-Unis y sont dépeints comme "l'autre» d'aujourd'hui, un malfaiteur récidiviste, cet article va plus loin du fait que la pertinence des leçons du passé est rattachée au groupe intérieur britannique actuel.

Ceci est visible dès le départ, où il ne s'agit pas simplement d'une critique de «ObamaBrown»: le groupe intérieur («nous») est dépeint comme "aimant chèrement [Kazai] » et « acceptant son élection totalement frauduleuse ». Même si l'on ne déduit pas de ce pronom personnel qu'il indique la satisfaction de la plus grande partie de la population par rapport à la position de son gouvernement, des usages ultérieurs de « notre » («notre aventure afghane actuelle ») mènent à percevoir le groupe intérieur comme co-responsable. C'est-à-dire qu'en racontant l' "aventure » comme étant « la nôtre » - indépendamment d'ailleurs du fait de savoir si c'était ou non l'intention de l'auteur -, le texte offre tout au moins la possibilité de relier le «nous » aux leçons des méfaits passés puisque «nous» prenons part à des méfaits dans le présent. Ceci est encore plus clair dans l'apogée dramatique de l'article, dans la phrase finale prophétique de Fisk: «notre mission occidentale en Afghanistan se soldera par un désastre absolu ». Ainsi, c'est "nous» (les Britanniques et l'Occident plus largement dont « nous » faisons partie), et non seulement les autres comme dans la rhétorique du

Argumentation et Analyse du Discours, 16 | 2016 
jugement, qui sommes présentés comme co-responsables des méfaits actuels, et qui sommes les destinataires de la leçon qui se dégage ici.

Ainsi, l'exemple illustre bien comment le topos de l'Histoire comme maître de la vie relie une représentation de "leur " méfait passé à la conclusion que "nous » aussi échouons actuellement; ou, pour être plus précis, qu'«un désastre absolu» nous menace si «nous » n'apprenons pas la leçon comme il se doit.

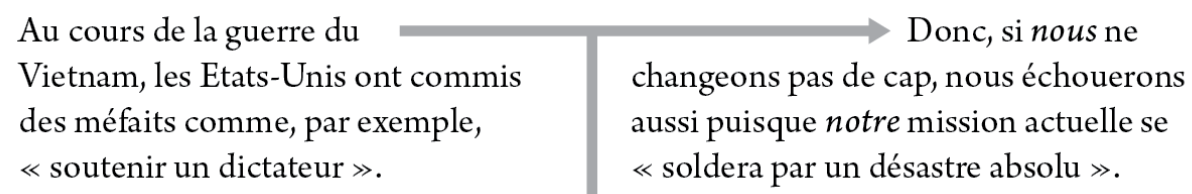

Du fait que l'histoire enseigne que « soutenir un dictateur » (et autres méfaits associés) est la mauvaise ligne de conduite, on devrait éviter de la suivre.

Figure 5 : La rhétorique de l'échec de Fisk.

\subsection{La rhétorique de la repentance}

Je passe maintenant à une conceptualisation de la rhétorique de la repentance. Je me baserai ici principalement sur des découvertes effectuées dans le champ interdisciplinaire des études de la mémoire, qui a prospéré en partie grâce à l'accroissement des «rituels publics d'aveux de culpabilité » autocritiques de ces dernières décennies ${ }^{11}$.

Dans la rhétorique de la repentance, de tels aveux (données) justifient les déclarations selon lesquelles les leçons ont bien été apprises; celles-ci sont couplées à des appels à nous confronter sans cesse à "nos " méfaits passés (conclusion). Le moi contemporain est donc imaginé comme se démarquant des actes coupables commis dans le passé (et des tentatives d'étouffer ce méfait), (Figure 6). En d'autres termes, un passé intériorisé est rejeté afin de construire un "autre" interne; dès lors, aucune rédemption définitive n'est possible. Cependant, si la «leçon» est connue, et que tirer l'enseignement du passé est représenté comme un processus continu dans et par lequel " nous » définissons ce que «nous " ne voulons pas être en regard de «notre " passé, cette démarche ne contredit pas nécessairement une image de soi quelque peu positive, dans le processus qui permet de faire advenir un sujet réformé (d'ailleurs, il parait impossible d'imaginer un acteur dont l'image de soi serait entièrement négative). 
\begin{tabular}{l|l}
$\begin{array}{l}\text { Nous sommes responsables } \longrightarrow \text { Ainsi, les leçons tirées } \\
\text { de méfaits passés. }\end{array}$ & $\begin{array}{l}\text { de l'histoire sont appliquées par notre } \\
\text { constante auto-surveillance, afin }\end{array}$ \\
d'empêcher la répétition de notre \\
échec passé.
\end{tabular}

Du fait que l'histoire enseigne que des actions spécifiques ont des conséquences spécifiques, on devrait accomplir ou omettre d'accomplir une action spécifique

dans une situation spécifique (prétendument) comparable à l'exemple historique auquel on se réfère.

Figure 6 : Une reconstruction de la rhétorique de la repentance.

Au sujet de récents aveux sur des méfaits passés, il est largement reconnu qu'à l'instar du Danemark et de la plupart des autres pays européens, la France d'après-guerre a vécu "pendant des décennies avec la difficulté d'admettre ce qui s'était réellement passé pendant la guerre, ainsi qu'avec le besoin irrésistible de bloquer la mémoire ou, tout du moins, de la refondre de manière plus utilisable » (Judt $2007: 808$ ). En France, cela concernait le "syndrome de Vichy" (Rousso 1994): il s'agissait d'occulter la collaboration entre le régime de Vichy, y compris ses politiques antisémites, et les occupants allemands. Ce «syndrome » évolua du mythe gaulliste d'une résistance quasi universelle, vers le silence qui régna pendant les présidences de Georges Pompidou et de Valéry Giscard d'Estaing, et le refus de François Mitterand de présenter des excuses (Golsan 2006: 78-84). Ce n'est que sous la présidence de Jacques Chirac que la France s'excusa officiellement, au $53^{\mathrm{e}}$ anniversaire de la rafle de 13.152 juifs au Vélodrome d'Hiver, en 1995. Néanmoins, et bien qu'il s'agisse d'une percée capitale, il a été suggéré que le discours de Chirac a rendu possible une affirmation de soi de la France (cf. l'analyse fascinante de Carrier 2005 : 74-80).

En 2012, au 70 anniversaire de la rafle, le Président français François Hollande, prononça un discours sur le site du vélodrome détruit qui illustre la mise en action de la rhétorique de la repentance (le premier paragraphe est tiré du début du discours, le second provient du milieu et le dernier de la partie finale du discours) :

Nous sommes rassemblés ce matin pour rappeler l'horreur d'un crime, exprimer le chagrin de ceux qui ont vécu la tragédie, évoquer les heures noires de la collaboration, notre histoire, et donc la responsabilité de la France.

La vérité, c'est que la police française, sur la base des listes qu'elle avait elle-même établies, s'est chargée d'arrêter les milliers d'innocents pris au piège le 16 juillet 1942. C'est que la gendarmerie française les a escortés jusqu'aux camps d'internement [...]

La vérité, c'est que pas un soldat allemand, pas un seul, ne fut mobilisé pour l'ensemble de l'opération.

La vérité, c'est que ce crime fut commis en France, par la France [...].

Notre vigilance ne doit jamais être prise en défaut. Aucune Nation, aucune société, aucune personne n'est immunisée contre le Mal. N'oublions pas ce jugement de Primo Levi à propos de ses persécuteurs: "Sauf exceptions, ils n'étaient pas des monstres; ils avaient notre visage ». Restons en alerte, afin de savoir déceler le retour de la monstruosité sous ses airs les plus anodins ${ }^{12}$. 

autour de l'échec tragique de la nation (en dépit des actions des «Justes ", du « Général de Gaulle ", de "la Résistance ", des "Forces françaises libres ", et d'« Institutions juives, telles l'œuvre de Secours aux Enfants - OSE ») à être à la hauteur de ses propres valeurs, principes et idéaux (la «laïcité», la «Liberté », la "Dignité humaine » et l'«Egalité et l'émancipation » sont mentionnées dans le texte ; par ailleurs, Hollande indique que «les Juifs étaient devenus des citoyens à part entière ", pour la première fois en Europe, en 1791 - ajoutant que « ce sont cette promesse et cette confiance qui furent piétinées il y a soixante-dix ans »). Tandis que Mikkelsen justifiait sa conclusion à l'aide d'une lecture positive de «nous » et de «nos » héros, Hollande évite de séparer une résistance héroïque et un petit groupe de fonctionnaires (par exemple) ayant collaboré avec l'Allemagne. Sur une telle toile de fond, la seule position que peut adopter la France contemporaine est celle d'une vigilance « héroïque » incessante.

Ceci se reflète clairement dans les choix linguistiques. Bien que le début du discours s'adresse aux personnes présentes, le genre utilisé indique que la désignation de l'acteur comme «nous » (sujet), «notre » et «nous » (complément) ne se limite pas à l'auditoire présent mais inclut la nation entière. On demande à cet acteur de réaliser de nombreuses choses, telles que «transmettre cette mémoire», d'exprimer son " chagrin », de rester "vigilant » et « en alerte »- toutes ces expressions signifiant que le passé est jugé négativement. L'événement lui-même, désigné sans aucun euphémisme, "l'horreur du crime», «la tragédie», «les heures noires de la collaboration, notre histoire ", est relié à la «France » elle-même, et explicitement désigné comme étant de "notre " responsabilité. Cette conclusion, basée sur ce que nous pouvons appeler le topos de la responsabilité (si quelqu'un commet quelque chose, il ou elle est responsable de ses conséquences), se fonde sur un certain nombre de raisons expressément citées. C'est ainsi qu'au début du discours, nous sommes face à une reconnaissance de responsabilité. Comme nous l'avons mentionné plus haut, le groupe intérieur adopte la position du coupable. Et de même que l'événement, leur rôle n'est pas relativisé. Ceci apparaît de façon évidente dans la qualification de la police et de la gendarmerie comme "françaises ", de même que dans l'insistance sur le fait que " pas un seul [soldat allemand n'a dû être mobilisé] ». Ce second paragraphe constitue peut-être la partie la plus explicite de tout le discours, et l'utilisation de l'anaphore (l'usage répété de "la vérité, c'est ») jette une lumière crue sur le méfait. De façon similaire, la référence à Primo Levi constitue un argument d'autorité, qui n'est pas selon moi un appel fallacieux à l'autorité ${ }^{13}$. En effet, il ne s'agit pas du seul support de l'argumentation globale de Hollande, et il se garde de traiter l'information sans équivoque de façon à ne pas offrir une solution claire et nette (comme si les méchants étaient des monstres et donc ontologiquement différents de «nous»). Au contraire, l'argument soulève des questions délicates pour le groupe intérieur contemporain.

Dans le même ordre d'idées, le texte ne vise pas un "autre » extérieur, mais bien un «autre " intérieur; en conséquence, Hollande appelle à faire des commémorations, à enseigner l'Holocauste, et à lutter contre l'antisémitisme en France. Bien que ce discours n'inclue aucune offre de réparation, il illustre la rhétorique de la repentance en endossant la responsabilité, en reniant l'acte, et en appelant à son interdiction. En résumé, comme illustré par la Figure 7, l'Historia Magistra Vitae conduit à une conclusion très différente de qui ressortait dans le cas de Mikkelsen. Ici, les données sont la représentation sans ambigüité - et non la tentative de l'étouffer - du passé coupable du 
groupe intérieur, qui justifie la conclusion que "nous" ne devons jamais laisser prendre notre vigilance en défaut.

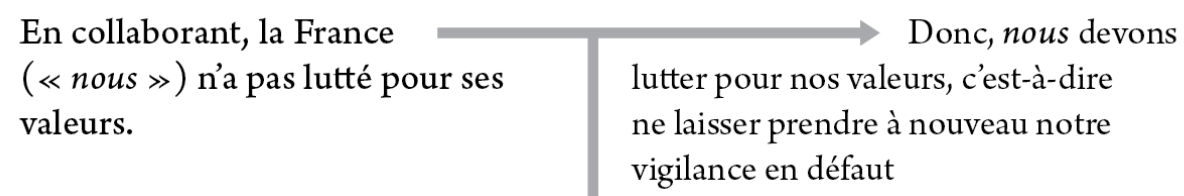

Du fait que l'Histoire enseigne que ne pas lutter pour ses valeurs peut avoir des conséquences terribles, nous devons nous garder de le faire.

Figure 7 : La rhétorique de la repentance de Hollande.

\subsection{La rhétorique du jugement-pénitence}

Au-delà de ces trois modes et de la perception généralisée du "mauvais silence " opposé à "de bons aveux sur de mauvaises actions ", je me propose maintenant de présenter une manière contre-intuitive d'utiliser les déclarations où l'on affirme avoir assimilé la leçon du passé. C'est-à-dire que «nous» avouons «notre» faute, afin de «nous » montrer moralement supérieurs à un «autre» extérieur (conclusion). Cette fois-ci, j'ai recours à un personnage du dernier roman publié par Camus, La Chute (1956) : un juge-pénitent. La devise de ce dernier, « plus je m'accuse moi-même, plus j'ai le droit de vous juger ", indique à quel point la légitimité grandissante conférée par la confession de ses propres méfaits permet de projeter une image du groupe intérieur en tant que pécheur repentant qui peut, implicitement ou explicitement, affirmer qu'il a appris les leçons du passé, et qu'il est devenu par-là moralement supérieur. En conséquence, les «autres » peuvent être représentés comme moralement inférieurs, précisément parce qu'une attitude humble envers « notre » passé douloureux «nous » élève au-dessus d'《eux » ${ }^{14}$.

41 Aussi bien Kindt que Reisigl et Wodak ont montré les aspects de la légitimation de soi qu'autorise l'Historia Magistra Vitae. Cette observation semble particulièrement pertinente en ce qui concerne la rhétorique du jugement, mais je voudrais avancer que la rhétorique du jugement-pénitence qui, à proprement parler, met en œuvre deux garanties, va plus loin. Outre le topos de l'Histoire comme guide de la vie, son impact provient d'une structure judéo-chrétienne initiale, de nos jours sécularisée, et qui peut se résumer par une parabole de Jésus destinée à ceux qui sont persuadés de leur propre bonté : « celui qui se glorifie sera humilié, et celui qui s'humilie sera glorifié » (Luc 18, 24). Je ne parle pas ici des aspects théologiques de cette citation, mais du fait qu'en raison de cette structure culturelle (un topos!), avouer ses propres méfaits passés confère au pénitent une légitimité. Dans un deuxième temps, cette légitimité est «utilisable » - et c'est exactement ce qui arrive dans la rhétorique du jugementpénitence (Figure 8). Sans doute, une telle légitimité peut-elle aussi se construire dans le cas de la rhétorique de la repentance ; néanmoins, c'est seulement ici qu'elle justifie en fin de compte de « rendre autre » un « autre » extérieur. 
Nous sommes responsables d'un méfait passé.
Ainsi, nous pouvons

légitimement nous revendiquer d'un domaine moral supérieur, et juger les autres pour ne pas avoir appris les leçons du passé.

Du fait que l'Histoire enseigne que des actions spécifiques ont des conséquences spécifiques, on devrait accomplir ou omettre d'accomplir une action spécifique dans une situation spécifique

(prétendument) comparable à l'exemple historique auquel on se réfère.

Figure 8 : Une reconstruction de la rhétorique du jugement-pénitence. comme maître et guide de la vie, ce qui exige, comme que nous le verrons, une
contextualisation moindre que dans les exemples précédents. Il est tiré de l'épilogue
d'un livre sur l'Europe et les États-Unis, rédigé par le politicien et avocat suisse Gret
Haller (2003: 227) et publié par un éditeur allemand. L'ouvrage traite des diverses
relations à l'État, la nation et la religion - au moyen desquelles l'auteur expose
prioritairement ses expériences en ex-Yougoslavie.

L'Europe a une histoire si longue et si chargée de culpabilité, non seulement au sein de son propre continent mais aussi dans les territoires colonisés d'autres continents, qu'elle a dû - et sans nul doute après la Seconde Guerre Mondiale redémarrer sans plus avoir la prétention d'effectuer un travail missionnaire auprès d'autres pour implanter ses propres convictions. Si les Européens veulent participer à des projets d'aide et de développement, une autre raison encore les empêche de considérer qu'une seule approche (la leur) est possible et juste. C'est l'expérience du pluralisme européen interne. Il n'en va pas de même pour les Américains des ÉtatsUnis, qui ont un sens profond de leur mission [Sendungsbewusstsein].

A première vue, ce qu'indique Haller semble refléter le discours de Hollande. Après tout, le passé de l'Europe n'est pas présenté en termes héroïques ou glorieux, ni ne passe sous silence "notre " méfait. Celui-ci est au contraire énoncé en toutes lettres. Cependant, il y a ici déviation par rapport à une description autocritique du passé. De fait, l'« Europe » devient un acteur qui regarde son sombre passé en face, et en émerge comme réformé, corrigé. Dès lors, elle peut maintenant confronter un autre extérieur, un nouveau méchant : les États-Unis d'Amérique.

Concernant la désignation du groupe intérieur, le manque de pronoms personnels tels que «nous» (en comparaison avec les cas précédents) est évident. Même si cela provient de la différence des genres, il est intéressant d'en constater l'effet : tandis que le «nous» donne au lecteur la possibilité de se désengager de la responsabilité collective, cette option n'existe plus guère lorsqu'on parle de l'« Europe » et des «Européens » - ce qui rend la construction très rigide. Dans la même veine, l'autre est désigné comme « les Américains des États-Unis »- sans aucune atténuation, rendant impossible toute différenciation. L'argument fallacieux de la généralisation hâtive est ainsi commise, avec pour effet d'établir une ligne de démarcation nette entre les acteurs. La nomination des événements et des processus (le colonialisme, « la Seconde Guerre Mondiale ») accentue la caractérisation initiale que Haller donne de l'Europe, comme dotée d'une «longue histoire chargée de culpabilité ». En se servant d'un 
argument fallacieux que l'on pourrait nommer argument par nécessité (si quelque chose a été exceptionnel, cela doit forcément avoir certaines conséquences), Haller stipule, sans aucun débat, que l'« Europe » « devait redémarrer », et qu'elle avait perdu son zèle missionnaire. Il faut noter que ceci n'est pas simplement une déclaration normative, mais est présenté comme un résultat (cette interprétation semble justifiée par le « une autre raison encore " présent dans la phrase suivante). Par contraste avec l'avertissement de Hollande de ne pas perdre sa vigilance, pour Haller, il semblerait que le travail a déjà été accompli puisque l'« Europe » est présentée comme réformée.

Dans le prédicat de l' " autre », le Sendungsbewusstsein (le "sentiment d'une mission ») allemand est un terme péjoratif, qui se réfère souvent au fanatisme religieux. Il contribue ainsi à la construction de l'«autre " comme manquant de civisme, et certainement comme très différent. Et effectivement, Haller poursuit son argumentation à la page suivante en se référant à la religion, déclarant que de « larges parties du public des États-Unis » tiennent des convictions absolues. Ce qui importe ici, ce n'est pas tellement de démontrer si la chose est vraie dans les faits (premièrement, et de même que les affirmations sur les «bonnes leçons", la religion peut aussi être mobilisée - avec de bonnes raisons - en faveur de conclusions très différentes; deuxièmement, le degré de sécularisation varie énormément au sein même de l'Union Européenne, et n'est pas toujours en avance sur celui des États-Unis (Baldwin 2009: 163-172). Ce qui importe, donc, c'est que l'affirmation est renforcée par l'usage de "profond» ("un sens profond de leur mission»), ce qui, une fois de plus, élargit la faille entre l'Europe et les « Américains des États-Unis ».

Pour résumer, l'utilisation du topos de l'Histoire comme maître de la vie mène ici à la conclusion que "nous» sommes des sujets réformés, alors que ce processus d'apprentissage "leur» manque à «eux» - une conclusion garantie à la fois par l'Historia Magistra Vitae, et par le modèle culturel selon lequel « celui qui se glorifie sera humilié, et celui qui s'humilie sera glorifié ». En mettant au premier plan «la longue histoire chargée de culpabilité ", c'est-à-dire un aveu des méfaits passés, Haller génère une légitimité qu'il exploite pour construire la supériorité du groupe intérieur par rapport à un autre moralement appauvri, et à ses actions.

\begin{tabular}{|c|c|}
\hline Nous admettons que & Ainsi, nous sommes \\
\hline$l^{\prime} \ll$ Europe $\gg$ a une $\ll$ longue & moralement supérieurs aux « Américains \\
\hline Histoire chargée de culpabilité ». & $\begin{array}{l}\text { des Etats-Unis » qui, eux, n’ont pas tiré la } \\
\text { leçon du passé. }\end{array}$ \\
\hline
\end{tabular}

Puisque l'Histoire enseigne qu'il faut regarder les méfaits passés et s'en souvenir afin de progresser moralement, on doit le faire.

Figure 9 : la rhétorique de Haller du jugement-pénitence. 


\section{Conclusion, ou : quels usages critiques du topos de l' Historia Magister Vitae?}

47 Je suis parti de l'hypothèse que les controverses discursives se basaient de plus en plus sur les interprétations de méfaits passés, plutôt que sur les perceptions de progrès futurs. Je me suis donc tourné vers l'Historia Magistra Vitae, et j'ai conceptualisé quatre utilisations de ce topos (la rhétorique du jugement, de l'échec, de la repentance et du jugement-pénitence), qui imaginent toutes des passés, des présents et des futurs particuliers. Ce faisant, j'ai accentué la logique du récit; la narrativisation du passé rend impossible toute représentation objective. Ceci ne signifie pas que les affirmations concernant la vérité d'événements particuliers échappent à l'évaluation - par exemple, les déclarations au sujet de ce qui est arrivé le 9 novembre 1989. Toutefois, cela signifie que le passé, dans la mesure où il est narrativisé, ne doit pas constituer la (seule) base d'évaluation de discours en général, et du topos de l'Histoire en tant que maitre et guide, en particulier.

A titre d'exemple, on pourrait d'un point de vue historiographique remettre en cause l'argument de Mikkelsen en disant que son parti participait d'un gouvernement qui a collaboré, qu'en tant que membre de ce gouvernement il a inconstitutionnellement interné des communistes danois, et que le soulèvement de 1943 n'avait pas les conservateurs pour origine. Historiquement parlant, cette critique serait exacte; cependant, on peut se poser la question de savoir jusqu'à quel point une telle perspective positiviste est appropriée lorsqu'on traite de structures culturelles profondes et de narratifs (nationaux). Il en va de même lorsqu'on aborde la rhétorique du jugement-pénitence, les structures culturelles dont elle s'inspire et les débats sur, disons, le fait de savoir s'il existe des différences entre les Etats-Unis et l'Europe, lesquelles et à quel point elles sont importantes, et si l'Europe a effectivement redémarré sur de nouvelles bases. Quoi qu'il en soit, la rhétorique du jugement et celle du jugement-pénitence, par leur structure culturelle, sont susceptibles de ne permettre qu'un traitement extrêmement limité d'informations.

$49 \mathrm{Au}$ lieu de se fonder sur l'historiographie, ou de rejeter l'argument analysé en luimême, la critique de ces rhétoriques pourrait consister à déceler le genre de préjugé qui empêche l'orateur aussi bien d'équilibrer son argumentation que de prendre une distance critique par rapport à son propre argument ${ }^{15}$. En d'autres termes, la forte cohérence narrative - Jérôme Bruner (1991: 9) parle de "séduction narrative " caractérisant la plupart des récits qui renforcent le soi face à un "autre » extérieur négatif, empêche le traitement inclusif et ouvert des arguments avancés ${ }^{16}$. Bien sûr, la rhétorique de l'échec et celle de la repentance proposent, elles aussi, des représentations particulières. Elles tendent cependant à mettre en cause leurs propres "préjugés» en soulevant des questions autocritiques au sujet du passé et/ou (crucialement) du présent de la communauté, permettant ainsi une reconstruction plus inclusive et ouverte des frontières symboliques actuelles du groupe intérieur. Ceci rappelle une brève note de Jürgen Habermas (1998:10), qui disait qu'apprendre à partir du passé, pour peu que cela soit possible, est un processus qui ne peut de nos jours être déclenché que par des déceptions, et par l'admission d'échecs passés. Par conséquent, l'aveu cognitivement exigeant de ses propres méfaits passés (ou même la reconnaissance explicite qu'un méfait passé - fût-il commis par quelqu'un d'autre constitue un avertissement pour le groupe intérieur actuel) peut devenir un instrument 
d'édification de soi, en ouvrant l'horizon du groupe intérieur aux expériences de l'« autre ». La confrontation aux souffrances de l'autre dont "nous» étions, sommes, ou pourrions être responsables, et à la reconnaissance de méfaits appartenant (potentiellement) au groupe intérieur, peut causer un décentrement du point de vue de ce dernier. Narrer de cette manière notre passé peut devenir la base de processus collectifs d'apprentissage ${ }^{17}$.

Ceci étant posé, et pour revenir aux quatre types conceptualisés ci-dessus, les rhétoriques du jugement et du jugement-pénitence réduisent au silence le doute intérieur qui offre un moteur d'apprentissage lorsque sont revendiquées des identités contemporaines entièrement constituées (évidemment plus fortement dans le cas des rhétoriques du jugement que dans celles du jugement-pénitence, puisque ces dernières englobent les mémoires de "notre " échec). Par conséquent, les processus collectifs d'apprentissage tendent à être bloqués. Par contraste, les rhétoriques de l'échec et de la repentance évitent cet écueil du fait qu'elles sont attentives aux voix des « autres » et à «notre " propre gêne (ceci est plus pertinent pour le cas de la rhétorique de la repentance, comparées à celles de l'échec, car elle vise le méfait passé et potentiellement présent du groupe intérieur). Le sujet narré demeure ici, au moins partiellement, fragmenté; ainsi les processus collectifs d'apprentissage sont-ils susceptibles d'être débloqués. Outre sa contribution, par la mise en place d'une taxonomie exhaustive, à l'étude des modalités selon lesquelles le topos de l'histoire comme maître et guide de la vie agit dans les débats publics, cette façon de voir offre un avantage supplémentaire : elle permet de considérer les emplois du topos en termes de mécanismes sociaux qui bloquent/débloquent des processus d'apprentissage collectifs, contribuant ainsi à tracer des frontières symboliques plus ouvertes/fermées.

51 Au-delà de la critique de ces théories - que je n'ai pu aborder ici que succinctement ces quatre types ne sont pas aussi nettement dissociés que pourrait le suggérer ma conceptualisation. Les textes sont des entités hétérogènes, qui incluent des arguments variés pouvant justifier chez les sujets des positions différentes. Ceci est bien illustré par Fisk, mais aussi par Hollande, dont les phrases finales - «C'est en étant lucides sur notre propre histoire que la France, grâce à l'esprit de concorde et d'union, portera le mieux ses valeurs, ici et partout dans le monde. Vive la République ! Vive la France !»glisse de la rhétorique de la repentance à celle du jugement-pénitence. Je ne vois aucun inconvénient à la référence positive faite à la France et à son esprit - comme je l'ai déjà dit, on ne peut s'attendre à ne trouver que des constructions de soi négatives. Néanmoins, en se tournant vers l'extérieur («promouvoir ses valeurs [...] dans le monde entier »), l'aveu de méfaits effectué par Hollande court le risque de rendre (implicitement) "autres" les "autres» extérieurs comme étant moins valables moralement ${ }^{18}$. En raison de la construction linguistique sans équivoque d'un "nous " culpabilisé, des exhortations vigoureuses à la vigilance, et du caractère imprécis de cette dernière phrase, je doute cependant que beaucoup puissent comprendre ce discours comme un appel à une supériorité morale. Néanmoins, ce détail final illustre le fait qu'à une époque où le passé est omniprésent, la mobilisation de sa "signification morale » dans des controverses publiques doit être minutieusement examinée. 


\section{BIBLIOGRAPHY}

Aristote. 2003 [1991]. Rhétorique (Paris : Gallimard)

Assmann, Jan. 1997. Moses the Egyptian. The Memory of Egypt in Western Monotheism (Cambridge : Harvard University Press)

Baldwin, Peter. 2009. The Narcissism of Minor Differences. How America and Europe are Alike (Oxford: Oxford University Press)

Barkan, Elazar \& Alexander Karn (éds). 2006. Taking Wrongs Seriously. Apologies and Reconciliation (Stanford : Stanford University Press)

Barthes, Roland. 1977. « Introduction to the structural analysis of narratives », Image, Music, Text (London : Fontana Press), 79-124

Birkelund, Peter. 2003. « Samarbejde eller brud? Det Konservative Folkepartis store dilemma », Joachim Lund (éd.). Partier under Pres - Demokratiet under Besaettelsen (Copenhagen : Gyldendal), 95-129

Bruner, Jerome. 1991. « The narrative construction of reality », Critical Inquiry 18, 1-21

Camus, Albert. 1956. La chute (Paris : Gallimard)

Carrier, Peter. 2005. Holocaust Monuments and National Memory Cultures in France and Germany since 1989 (New York : Berghahn)

Cicero. 1959. De Oratore. Books I \& II (London : Heinemann)

Cunningham,Michael.(1999) « Saying sorry: the politics of apology », Political Quarterly 70, 285-293

DIIS (2005), Danmark under den kolde krig. Den sikkerhedspolitiske situation 1945-1991. Bind 2: 1963-1978 (Copenhagen : DIIS)

Eder, Klaus. 1999. « Societies learn and yet the world is hard to change », European Journal of Social Theory 2, 195-215

Farbøl, Rosanna. 2011. « Irakkrigen og den historiske ulegitimering », Slagmark. Tidsskrift for Idéhistorie 60, 73-85

Fisk, Robert. 2009. « America is performing its familiar role of propping up a dictator ", The Independent, 4 November 2009, http://www.independent.co.uk/voices/commentators/fisk/ robert-fisk-america-is-performing-its-familiar-role-of-propping-up-a-dictator-1814194.html Forchtner, Bernhard. 2013. « Nazi-collaboration, acknowledgements of wrongdoing and the legitimation of the Iraq war in Denmark : a judge-penitent perspective », Piotr Cap \& Urszula Okulska (éds). Analyzing Genres in Political Communication : Theory and Practice (Amsterdam : John Benjamins), 239-265

Forchtner, Bernhard. 2014. « Rhetorics of judge-penitence: claiming moral superiority through admissions of past wrongdoing ", Memory Studies 7, 409-424

Forchtner, Bernhard \& Christoffer Kølvraa, C. 2012. « Narrating a 'new Europe' : from 'bitter past' to self-righteousness ", Discourse \& Society $23,377-400$

Gibney, Marc, Rhoda E. Howard-Hassmann, Jean-Marc Coicaud \& Niklaus Steiner (éds). 2008. The Age of Apology. Facing up to the Past(Philadelphia : University of Philadelphia Press)

Giesen, Bernhard. 2004. Triumph and Trauma (Boulder : Paradigm) 
Golsan, Richard. J. 2006. « The legacy of World War II in France. Mapping the discourses of memory ", Richard Ned Lebow, Wulf Kansteiner \& Claudio Fogu (éds). The Politics of Memory in Postwar Europe (Durham : Duke University Press), 73-101

Greimas, Algirdas J. 1966. Sémantique structurale. Recherche de méthode (Paris : Larousse)

Greimas, Algirdas J. 1987. On Meaning. Selected Writings in Semiotic Theory (Minneapolis : University of Minneapolis Press)

Habermas, Jürgen. 1998. “Can we learn from history?”, A Berlin Republic: Writings on Germany (Cambridge: University of Nebraska Press), 5-13

Habermas, Jürgen. 2004. Wahrheit und Rechtfertigung (Frankfurt/M. : Suhrkamp)

Halbwachs, Maurice. 1950. La mémoire collective (Paris : Albin Michel)

Haller, Gret. 2003. Die Grenzen der Solidarität. Europa und die USA im Umgang mit Staat, Nation und Religion (Berlin : Aufbau)

Heer, Hannes, Walter Manoschek, Alexander Pollak \& Ruth Wodak (éds). 2008. The Discursive Construction of History : Remembering the Wehrmacht's War of Annihilation (Palgrave : Basingstoke)

Hollande, François. 2012. Discours du Président de la République à l'occasion du $70^{\text {ème }}$ anniversaire de la rafle du Ve' d'Hiv, http://www.elysee.fr/declarations/article/discours-du-president-de-larepublique-a-l-occasiondu-70eme-anniversaire-de-la-rafle-du-vel-d-hiv/

Judt, Tony. 2007. Postwar. A History of Europe Since 1945 (London : Pimlico)

Kaae, Martin \& Jesper Nissen. 2008. Vejen til Iraq - Hvorfor gik Danmark i Krig ?(Copenhagen : Gads)

Kienpointer, Manfred. 1995. « Rhetoric », Jan Verschuren, J. O. Östman \& J. Blommaert (éds). Handbook of Pragmatics. Manual (Amsterdam : John Benjamins), 453-461

Kindt, Walter. 1992. «Argumentation und Konfliktaustragung in Äußerungen über den Golfkrieg », Zeitschrift für Sprachwissenschaft 11, 189-215

Kirchhoff, Hans. 2004. Samarbejde og Modstand Under Bescettelsen - En Politisk Historie(Odense : Syddansk Universitetsforlag)

Koselleck, Reinhard. 1999. Vergangene Zukunft. Zur Semantik geschichtlicher Zeiten (Frankfurt/M : Suhrkamp)

Maillat, Didier \& Steve Oswald. 2011. « Constraining context: a pragmatic account of cognitive manipulation ", Christopher Hart (ed.) Critical Discourse Studies in Context and Cognition (Amsterdam : John Benjamins), 65-80

Mikkelsen, Brian. 2003, « Aldrig mere i neutralitetens fælde », Berlingske Tidende, 30 août, p. 8

Miller, Max. 2002. Some Theoretical Aspects of Systemic Learning. http://www.sozialwiss.unihamburg.de/Isoz/isoz/miller/miller/systemic_learning.pdf

Müller, Jan-Werner. 2002. « Introduction », Memory and Power in Post-War Europe: Studies in the Presence of the Past (Cambridge : Cambridge University Press), 1-35

Musolff, Andreas. 2004. Metaphor and Political Discourse. Analogical Reasoning in Debates about Europe (Basingstoke : Palgrave)

Olick, Jeffrey K., Vered Vinitzky-Seroussi \& Daniel Levy (éds). 2011. « Introduction”, The Collective Memory Reader (Oxford : Oxford University Press), 3-62 
Reisigl, Martin. 1998. « 50 Jahre Zweite Republik - Zur diskursiven Konstruktion der österreichischen Identität in politischen Gedenkreden », Panagl, O. (ed.), Fahnenwörter in der Politik - Kontinuitäten und Brüche (Wien : Böhlau), 217-251

Reisigl, Martin. 2007. Nationale Rhetorik in Fest- und Gedenkreden. Eine diskursanalytische Studie zum „österreichischen Millennium“ in den Jahren 1946 und 1996 (Tübingen : Stauffenburg)

Reisigl, Martin. 2013. « Critical Discourse Analysis », Bayley, R., R. Camerron \& C. Lucas (éds). The Oxford Handbook of Sociolinguistics (Oxford : Oxford University Press), 67-90

Reisigl, Martin \& Ruth Wodak. 2001. Discourse and Discrimination: Rhetorics of Racism and antiSemitism(London : Routledge)

Reisigl, Martin \& Ruth Wodak. 2009. «The discourse-historical approach », Wodak, R. \& M. Meyer (éds). Methods of Critical Discourse Analysis(revised ed.)(London : Sage), 87-121

Ricoeur, Paul. 1991. Temps et Récit 3. Le temps raconté (Paris : Points)

Rousso, Henry. 1987. Le syndrome de Vichy (1944-1987) (Paris : Seuil)

Rubinelli, Sara. 2009. Ars Topica. The Classical Technique of Constructing Arguments from Aristotle to Cicero (Berlin : Springer)

Tilly, Charles. 2002. Stories, Identities, and Political Change (Oxford : Rowman \& Littlefield)

Toulmin, Stephen, Richard Rieke \& Allan Janik. 1979. An Introduction to Reasoning (New York : MacMillan)

van Dijk, Teun A. 1980. «Story comprehension: an introduction », Poetics 9 (1-3), 1-21

Viehöver, Willy. 2011. « Diskurse als Narration », Reiner Keller, Andreas Hirseland, Werner Schneider \& W. Viehöver (éds). Handbuch Sozialwissenschaftliche Diskursanalyse, vol. 1 (Wiesbaden : VS), 193-224

Walton, Douglas. 1991. « Bias, critical doubt, and fallacies », Argumentation and Advocacy 28, 1-22

Walton, Douglas, Chris Reed \& Fabrizio Macagno. 2008. Argumentation Schemes (Cambridge : Cambridge University Press)

Wengeler, Martin. 2003. « Argumentationstopos als sprachwissenschaftlicher Gegenstand. Für eine Erweiterung linguistischer Methoden bei der Analyse öffentlicher Diskurse », Susan Geideck $\&$ Wolf-Andreas Liebert (éds).Sinnformeln. Linguistische und soziologische Analysen von Leitbildern, Metaphern und anderen kollektiven Orientierungsmustern (Berlin \&New York : de Gruyter), 59-82 White, Hayden. 1980. « Narrativity in the representation of reality », Critical Inquiry 7, 5-27

Wodak, Ruth \& Rudolf de Cillia. 2007. « Commemorating the past: the discursive construction of official narratives about the Rebirth of the Second Austrian Republic », Discourse \& Communication $1,337-363$

Wodak, Ruth, Rudolf de Cilia, Martin Reisigl \& Karin Liebhart. 2009. The Discursive Construction of National Identity (Edinburgh : Edinburgh University Press)

Wodak, Ruth, Rudolf de Cillia, Martin Reisigl, Karin Liebhart, Klaus Hoffstätter \& Maria Kargl. 1998. Zur diskursiven Konstruktion von nationaler Identität (Frankfurt \& Main : Suhrkamp)

Wodak, Ruth, Florian Menz, Richard Mitten \& Frank Stern. 1994. Die Sprachen der Vergangenheit. Öffentliches Gedenken in österreichischen und deutschen Medien (Frankfurt \& Main : Suhrkamp) 
Wodak, Ruth, Peter Nowak, Johanna Pelikan, Helmut Gruber, Rudolf de Cilla \& Richard Mitten. 1990. Wir sind alle unschuldige Täter. Diskurshistorische Studien zu Nachkriegsantisemitismus

(Frankfurt \& Main : Suhrkamp)

\section{NOTES}

1. En m'inspirant de l'approche discursive-historique dans les études critiques de discours, j'aurais pu évoquer ces quatre structures de communication dans des termes de stratégies différentes de présentation de soi et de l'autre. Bien que la notion de rhétorique rappelle celle de stratégie (Reisigl et Wodak 2009: 94), j'ai néanmoins préféré le terme de rhétorique ici, du fait qu'il ne lie pas ma conceptualisation à un cadrage particulier. De plus, ces rhétoriques sont considérées comme diverses, le pluriel indiquant la variété de réalisations envisageables (cf. aussi Forchtner et Kolvraa 2012, Forchtner 2014).

2. $\mathrm{Au}$ prix de perdre la répétition voulue entre rhetorics of penitence et rhetorics of judgepenitence,nous traduisons le premier par rhétorique de la repentance, qui semble plus adéquat en français, et maintenons pour le second la notion de juge-pénitent avancée par Camus (Note de la traductrice).

3. Cf. parmi de nombreux autres, Heer et al. 2008, Wodak et al. 1990, 1994, 1998, 2009, Reisigl 2007, Wodak et de Cillia 2007.

4. Cette approche qui s'insère dans le courant anglo-saxon critique de l'analyse du discours (Critical Discourse Analysis ou CDA), est exposée entre autres dans Reisigl et Wodak 2009. 87-121.

5. Pour une bonne vue d'ensemble, cf. Olick et al. 2011.

6. Cf. Barthes 1977, Greimas 1983 et 1987 ; je ne suis pas en mesure ici de débattre de concepts pertinents mais on pourra consulter Viehöver (2011).

7. Cicéron 1969, II, LXXXVI.

8. Pour plus de détails, cf. Kirchhoff 2004, Kaae et Nissen 2008, Farbol, 201.

9. Parmi les quatre partis membres du gouvernement d'union nationale, et qui étaient donc responsables de la politique de coopération - les conservateurs, les libéraux, les sociaux-libéraux et les sociaux-démocrates - le parti de Mikkelsen était cependant le plus critique sur la collaboration (cf. Birkelund $2003: 119$ ).

10. C'est sur ce fond qu'une brève référence à «notre » méfait passé («l'armée coloniale britannique du XIXème siècle »), dans un paragraphe qui n'a pas été intégré ici, n'affecte pas le statut de la guerre des Etats-Unis au Vietnam comme étant «le» méfait passé duquel nous devons tirer des leçons.

11. Giesen $2004: 130$; pour des recueils de cas cf. Cunningham 1999, Barkan et Karn 2006, Gibney et al. 2008.

12. http://www.elysee.fr/declarations/article/discours-du-president-de-la-republique-a-loccasion-du-70eme-anniversaire-de-la-rafle-du-vel-d-hiv/

13. Pour les arguments d'opinion experte, cf. Walton et al. $2008: 15$.

14. Pour une explication plus détaillé sur le jugement-pénitence cf. Forchtner 2014.

15. Je me réfère ici à seulement deux des cinq caractéristiques proposées par Walton (1991: 19).

16. Pour comprendre comment agissent de tels processus, on peut se référer à la notion de manipulation comme l'entendent Maillat et Oswald (2011 : 71), qui impose l'interprétation d'une information «dans un contexte limité " et bloque "l'accès à toute hypothèse contextuelle alternative ", ainsi qu'à l'idée d'Andreas Musolff (2004: 173-177) sur les cas de figures métaphoriques ouverts et fermés. Tandis que les premiers sont ouverts à la critique, les derniers facilitent le verrouillage de la pensée.

17. Pour la notion de processus collectifs d'apprentissage, cf. parmi d'autres Eder 1999, Miller 2002 , Habermas 2003. 
18. Pour des cas similaires au niveau des États-Unis, cf. Forchtner et Kolvraa 2012.

\section{ABSTRACTS}

The idea that "the past" could provide guidance in the present is a popular topos in public debates. Being interested in how this topos of historia magistra vitae can be utilized in discourses, representations and their consequences, this article asks how claims to know 'the lessons' from the past, for having learnt, give meaning to contemporary actors, events, objects and processes. The article thus raises questions such as 'what pasts and presents does this topos draw on?' and 'what conclusions does the use of this topos in public struggles over demarcating 'us' from 'them' facilitate?'. Instead of providing a case study, this article offers an abstract typology of such claims by conceptualising four types of uses: rhetorics of judging, rhetorics of failing, rhetorics of penitence and rhetorics of judge-penitence. In so doing, the article carefully differentiates these uses and, thus, provides a framework for better understanding them across a range of cases.

L'idée que « le passé " puisse contribuer à mieux guider le présent est un topos populaire dans les débats publics. En recherchant la manière dont ce topos de la historia magistra vitae peut être utilisé dans des discours, des représentations et leurs conséquences, cet article cherche à voir comment des affirmations qui disent avoir appris «les leçons » du passé peuvent donner un sens à des acteurs, des événements, des objets et des processus contemporains. On pose donc des questions telles que «sur quels passés et quels présents ce topos se base-t-il ? » et «à quelles conclusions possibles accède-t-on dans l'utilisation de ce topos dans des luttes publiques sur la ligne de démarcation entre "eux" et "nous" ? Au lieu de proposer une étude de cas, cet article offre une typologie abstraite de telles affirmations, en conceptualisant quatre sortes d'utilisation: les rhétoriques de jugement, les rhétoriques de l'échec, les rhétoriques de repentance, et les rhétoriques de jugement-repentance. Ce faisant, il établit une distinction minutieuse entre ces diverses utilisations, et procure un cadre qui permettra de mieux les comprendre à travers une série de cas.

\section{INDEX}

Keywords: Critical Discourse Analysis (CDA), discourse-historical approach, historia magistra vitae, topos

Mots-clés: approche historico-discursive, Critical Discours Analysis (CDA), historia magistra vitae, topos

\section{AUTHORS}

\section{BERNHARD FORCHTNER}

Humboldt Universität zu Berlin 